\title{
A CASE REPORT
}

\section{A case report on Actinic cheilitis: a rarer entity among Sri Lankans}

\author{
D. R. D. L. Ratnayake, ${ }^{1,3}$, R. M. S. H. B. Medawela ${ }^{2}$, R. D. Jayasinghe ${ }^{2}$ and B. S. M. S. Siriwardena ${ }^{1}$ \\ ${ }^{1}$ Department of Oral Pathology, Faculty of Dental Sciences, University of Peradeniya, Peradeniya, Sri Lanka \\ ${ }^{2}$ Department of Oral Medicine and Periodontology, Faculty of Dental Sciences, University of Peradeniya, Peradeniya, \\ Sri Lanka \\ ${ }^{3}$ Ministry of Health, SUWASIRIPAYA, Baddegama Wimalawansa Thero Mawatha,Colombo 10, Sri Lanka
}

Received:26/10/2017; Accepted:22/02/2018

\begin{abstract}
Actinic keratosis (AK) is a dermatological condition commonly found among fair-skinned populations and considered as a potentially malignant disorder usually appearing on sunexposed areas such as the scalp, face and forearms. It is an uncommon disorder among Asians. Therefore, Actinic Keratosis in Asians has not been thoroughly investigated. AK is clinically termed as"Actinic chelitis" when lesions present in the lips. This case report is about a 42-year-old male who presented at the Oral Medicine Clinic of the University Dental Hospital, Peradeniya, with a complaint of burning sensation of the mouth which aggravates with spicy foods for about three months. Been working in a farm from the age of 12 years and exposure to sunlight for about 9 to 10 hours a day, he has had an extreme exposure to sunlight. Examination revealed an erythematous, nonindurated ulceration of the lower lip. Histopathological sections showed ulcerated mucosa covered by orthokeratinized stratified squamous epithelium with regenerative atypia towards the ulcer bed. Degenerative changes of collagen were evident in the upper corium. The patient was educated about the disease and a protective local covering agent was prescribed to apply on the affected area. This case report emphasizes the possibility of acquiring $\mathrm{AK}$ among dark-skinned people due to extreme exposure to sunlight and the importance of knowledge about this disease entity for clinicians when diagnosing as it may have been misdiagnosed.
\end{abstract}

Keywords: Actinic keratosis, Actinic chelitis, Asians, Solar keratosis, UV radiation.

\section{INTRODUCTION}

Actinic cheilitis (AC) is a clinical term for an ulcerative, sometimes crust-forming lesion of the mucosa of part or entire vermillion border of the lower lip (van der Waal, 2009). It is a potentially malignant disorder in which the histopathologic spectrum varies from hyperkeratosis with or without epithelial dysplasia to early squamous cell carcinoma in the presence of basophilic changes in the lamina propria. Thus, it is histopathologically termed as Actinic Keratoses (AK) (van der Waal, 2009; Vieira et al., 2012). Ultraviolet radiation is thought to be the major etiological factor, with age (due to diminished cellular DNA repair mechanisms), immunosuppression and tobacco use being considered as important contributing factors (Frost et al., 1998).

It is commonly seen in fair skinned populations. As this is not a common entity among dark skinned populations in eastern countries, AC in Asians has not been so thoroughly investigated. Herewith we are presenting a case of Actinic cheilitis in a 42 year old male, the first documented case of $\mathrm{AC}$ in Sri Lanka.

\section{CASE PRESENTATION}

A 42 year old male presented to the Oral Medicine Clinic, University Dental Hospital, Peradeniya, Sri Lanka, in April 2016 with a complaint of burning sensation of the mouth which aggravates with spicy foods for a period of three months. The patient gave a history of recurrent ulcerations which healed spontaneously leaving a crust in the lower lip. He has been working in farms since childhood, spending almost 9 - 10 hours in the field on daily basis. He is a betel chewer with all ingredients for a period of 6 years. The patient has a medical history of Gastro oesophageal reflux disorder and was on medication.

Extra oral examination revealed depigmentation of the vermillion borders of the lips with no associated skin lesions. He presented with an erythematous non indurated ulcers measuring $1 \mathrm{~cm} \times 0.3 \mathrm{~cm}$ with yellowish crusty surface involving the lower lip only (Figure 1). The patient also had a depapillated tongue.

Histopathological and heamatological investigations were carried out to arrive at a definitive diagnosis. Histopathology revealed an ulcerated mucosa covered by orthokeratinized stratified squamous epithelium with solar elastoses in the lamina propria (Figure 2) and regenerative atypia towards ulcer bed without marked dysplasia. Degenerative changes of collagen were evident in the upper corium. These histopathological features were consistent with those of Actinic Keratosis and confirmed the clinical diagnosis of actinic cheilitis.

The patient was educated about the condition and prescribed a protective local covering agent (Vasline -Topical application four times daily). The patient was

*Corresponding Author's Email: sumuduhimesha@gmail.com http://orcid.org/0000-0002-2714-689X This article is published under the Creative Commons Attribution License (https://creativecommons.org/licenses/by/4.0/), which permits unrestricted use, distribution, and reproduction in any medium, provided the original work is properly cited. 


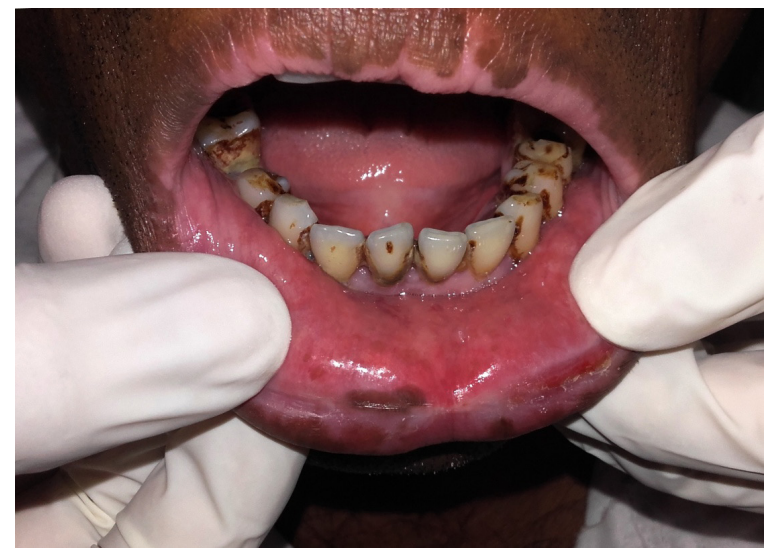

Figure 1: Ulcerated lower lip and Depapillated tongue.

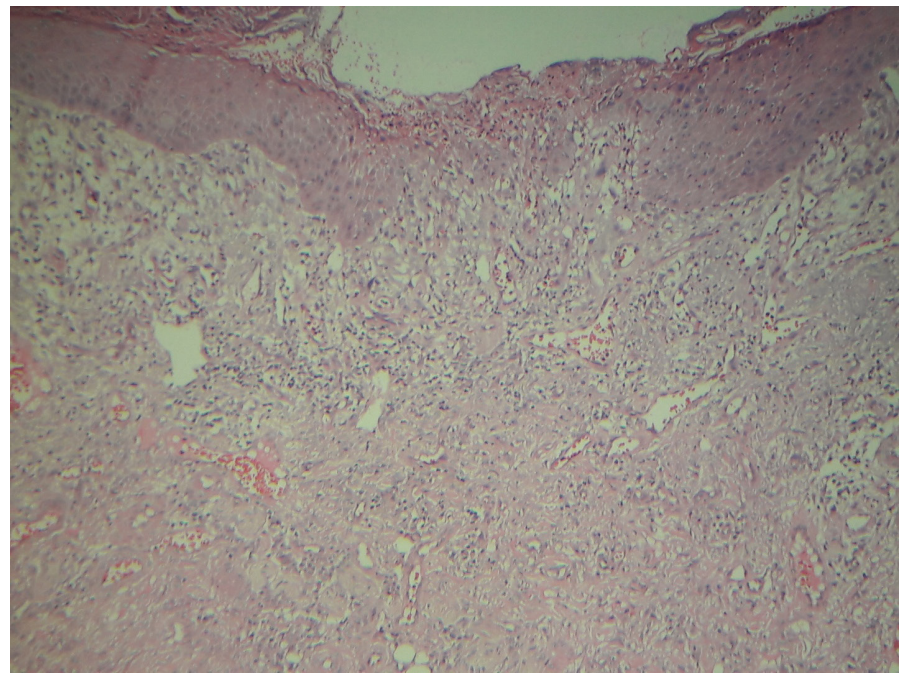

Figure 2: Orthokeratinized stratified squamous epithelium with solar elastoses in the lamina propria.

put on regular follow-up care with strong advices on habit cessation (Betel, Areca nut, Tobacco). After two years follow up, the patient was symptoms free with no signs of malignant transformation.

\section{DISCUSSION}

$\mathrm{AK}$ is a prevalent dermatological condition and has been widely studied in fair-skinned populations. Actinic cheilitis is the counterpart of actinic keratosis of the skin affecting oral mucosa. It has been recorded in parts of Australia and the southern part of United States. The lifetime risk of developing AK was higher than $50 \%$ and may reach 90 $\%$ among those who live to be 80 years or older (Frost et al., 1998). A study done among 328 cases of AK in eastern China revealed the mean age affected was $72 \pm 19.6$ years (range 39-93) and $88.4 \%$ of the patients (290 cases) were older than 60 years, and $65.2 \%$ (214 cases) older than 70 years. More than half of the patients were farmers $(51.8 \%$, 170 cases) (Han et al., 2013). The literature on the burden of the disease among dark-skinned Asians is scarce.

The incidence of AK is tightly linked to ultraviolet (UV) irradiation, as they are found on chronically sun-exposed skin such as face and dorsum of hands. Advancing age and lighter skin further increases the risk of developing lesions (Han et al., 2013). The classical profile reported in the literature for a patient at high risk for actinic cheilitis is a Caucasian man above 50 years of age, who abuses tobacco and has a chronic history of exposure to sunlight (van der Waal, 2009; Vieira et al., 2012; Han et al., 2013). Although our patient was in his middle age, he has been exposed to sunlight for a longer duration since his childhood and has a history of betel quid chewing with tobacco. The literature suggests that eversion of the lips increasing exposure of thin epithelium (with low keratin and melanin) as in current case further increases the risk of developing AC.

Actinic cheilitis may appear clinically with localized or diffused areas of white, red or mixed color lesions. Whitish areas represent hyperkeratotic lesions, whereas the erosive or ulcerated lesions confer cheilitis (Han et al., 2013). However, the clinical features including habit of areca nut chewing, depigmentation of the lips, depapillation of the tongue were strongly suggestive of early oral sub mucous fibrosis which is a prevalent oral potentially malignant disorder in Sri Lanka. Therefore, there is a high chance to miss AC in the list of differential diagnosis of a less experienced clinician as knowledge on AK might be inadequate due to rarer incidence in our part of the region in the world.

Histopathologically, AKs are proliferations of transformed, neoplastic keratinocytes confined to the epidermis. Additionally, basophilic degeneration of collagen fiber which is termed as "solar elastosis" can be seen in connective tissue. These lesions are currently termed 
AKs while they remain confined to the epidermis, but when they extend more deeply to involve the papillary and/or reticular dermis, they are termed squamous cell carcinoma (SCC) (Han et al., 2013). AKs and SCC represent the same disease process at different stages of evolution. The present case showed no dysplasia except regenerative atypia due to ulceration and solar elastosis in the upper part of connective tissue corium. Basophilic changes of the connective tissue are a constant finding in actinic cheilitis which occurs due to replacement of collagen by an amorphous basophilic material (Han et al., 2013). Although it is agreed that AK may progress to become invasive cutaneous squamous cell carcinoma (SCC), the frequency of this occurrence and the biologic behavior of the resultant lesion remain debatable. Some literature reports the risk of progression to SCC is as $0.025 \%$ - $20 \%$ /year / lesion (van der Waal, 2009).

The interventions available for the treatment of $\mathrm{AK}$ include surgical and non surgical methods. Surgical methods include excision, cryosurgery, curettage and laser surgery. Non surgical treatments include topical chemotherapy and dermabrasion. According to the systematic review on interventions for AK it is concluded that for individual lesions, photodynamic therapy appears more effective and has a better cosmetic outcome than cryotherapy (Roewert-Huber et al., 2007). As current lesion did not show marked dysplasia histopathologically, surgery was not considered as a management option. Case was discussed in multidisciplinary management clinic and considering availability of facilities management was limited to continuous monitoring and application of topical covering agent, deviating from standard care.

\section{CONFLICT OF INTERESTS}

The authors certify that they have no affiliation or involvement with any organization or entity with any financial interest (such as honoraria; educational grants; participation in speakers' bureaus; membership, employment, consultancies, stock ownership, or other equity interest; and expert testimony or patent-licensing arrangements), or non-financial interest (such as personal or professional relationships, affiliations, knowledge or beliefs) in the subject matter or materials discussed in this manuscript. The authors declare that there is no conflict of interest regarding the publication of this paper.

\section{REFERENCES}

Frost, C.A., Green, A.C. and Williams, G.M. (1998). The prevalence and determinants of solar keratoses at a subtropical latitude (Queensland, Australia). British Journal of Dermatology 139: 1033-1039.

Han, Y.M., Chen, L.J., Dou, X. and Yang Q.P. (2013). Clinical and pathological study of 328 cases of actinic keratosis in eastern Chinese patients. Dermatology 227(4): 316-20.

Roewert-Huber, J., Stockfleth, E. and Kerl, H. (2007). Pathology and pathobiology of actinic (solar) keratosis - an update. British Journal of Dermatology 157: 1820.

van der Waal, I. (2009). Potentially malignant disorders of the oral and oropharyngeal mucosa; terminology, classification and present concepts of management. Oral Oncology 45(4-5): 317-23.

Vieira, R.A., Minicucci, E.M., Marques, M.E. and Marques, S.A. (2012). Actinic cheilitis and squamous cell carcinoma of the lip: clinical, histopathological and immunogenetic aspects. Anais Brasileiros de Dermatologia 87(1): 105-14. 